\title{
Recovering Dusty Shoe Prints from Skin: Comparative Research
}

\author{
Yaron Shor ${ }^{*}, 1$, Amit Cohen ${ }^{2}$, Sarena Wiesner ${ }^{1}$, and Revital Weiss ${ }^{1}$ \\ ${ }^{1}$ Toolmarks and Materials Laboratory, DIFS, Israel Police H.Q., Jerusalem, Israel \\ ${ }^{2}$ Serious Crime Mobile Laboratory, DIFS, Israel Police, Israel
}

\begin{abstract}
Dusty shoeprints can be found on various substrates and should be dealt with properly. When the shoeprints are located on a human body the challenge is greater, due to the curved shape of the human body and the skin texture.

We made several dust impressions on a human volunteer. The prints were lifted using three different methods: electrostatic lifter, black gelatin lifter and white adhesive lifter followed by enhancement with Bromophenol Blue (BPB).

The prints recovered on the white adhesive were screened by skin debris that reacted with the Bromophenol blue. Full contact was not achieved between curved organs and the ESL. Another disadvantage of the ESL is that the electrostatic charge might be dangerous when used on live subjects. The best method was found to be the black gelatin lifter.
\end{abstract}

We recommend using gelatin lifter for lifting dust shoeprint from live bodies.

Keywords: Forensic science, shoeprints, footwear impression, electrostatic dust print lifter, gelatin lifter, adhesive lifter, Bromophenol blue, dust print, skin.

\section{INTRODUCTION}

Many shoeprints can be found at crime scenes, yet not all of them may be directly connected to the crime committed. If shoeprints are found on corpses or live victims, they are of potential significance, since they well may have been left by the perpetrator, thus being directly linked to the crime. Such shoeprints can result from the perpetrator's accidentally having stepped on the victim during commission of the crime or while fleeing; alternatively, there have been cases in which the perpetrator intentionally stomps on the victim, producing shoeprints on the skin or on clothing. These shoeprints are usually blunt force injuries ${ }^{1}[1,2]$ or those in which some material, such as blood, mud, grease or dust [3, 4], is transferred from the shoe to the skin.

High resolution photographs with optimal illumination must be taken prior to any attempt at the crime scene or in the laboratory for further examination. Photographing prints on bodies is challenging, because of the curvature of most organs and difficulties in placing the scale properly to capture good depth of field.

The second step is to identify the material that produced the shoeprints. Blunt force injuries are defined by blood accumulating under the skin, creating some permanent color changes. Extraneous materials (e.g., blood, grease or dirt)

*Address correspondence to this author at the Toolmarks and Materials Laboratory, DIFS, Israel Police H.Q., Jerusalem 91906, Israel; Tel: 972-25429353; Fax: 972-2-5898078; E-mail: simanim@police.gov.il

\footnotetext{
${ }^{1}$ Van Dijk, T.M. Case study: Two distinctly different shoe outsole patterns, impressed on the body of a young male, used to implicate both suspects in his death. Abstract, International Symposium on the Forensic Aspects of Footwear and Tire Impression Evidence; FBI Academy, 1994.
}

adhere to the outer layer of the skin and create a color stain on the skin. The color of this impression is derived from the material that created it. Sometimes it can be confusing to determine if the mark is interior or exterior. The answer can be determined with a gentle wipe of a small corner of the shoeprint.

After identifying the source of the shoeprints, the most appropriate method of enhancing must be determined. The only perpetuation method for blunt force shoeprints is photography. Internal blood accumulation becomes darker after force has been exerted. On cadavers the blood remains in situ, and marks appear more intense as time passes. It is recommended to photograph such prints again after several hours [2].

Blunt force shoeprints behave differently on live persons. Blood can move to other places with the natural blood flow of the body, and therefore photographs must be taken as close to the time of the event as possible.

Shoeprints created by extraneous materials can be lifted and chemically enhanced using the same methods commonly applied on other surfaces.

Latent shoeprints can appear when fingerprint enhancing methods are applied $[3,5]$.

Shoeprints consisting of blood will usually be brownishred. A test with a blood identifying reagent such as Hexagon can be used to identify blood with greater certainty. Such shoeprints should be further enhanced with blood reagents [6] or even lifted prior to enhancement [7].

Three commonly used methods for lifting dust shoeprints are electrostatic lifters [8], gelatin lifters [9], and an adhesive lifter followed by a chemical enhancement. Bromophenol blue $(\mathrm{BPB})$ is a chemical reaction that has been used 
successfully [10]. Several researches were performed ${ }^{2}[11]$ comparing electrostatic lifter and gelatin lifer, but none of them compared their performance on bodies.

$\mathrm{BPB}$ is a $\mathrm{pH}$ indicator with a $\mathrm{pH}$ transition range of 3-4.6 [12]. After spraying the adhesive with $\mathrm{BPB}$, the dust reacts with the BPB in the reduced form, a transition occurs, and the dust particles change their color to blue.

An example of dry origin shoeprints that appeared on a body occurred in a murder case several years ago. The body of a female prostitute was found with a dry origin shoeprint on her abdomen (Fig. 1). No comparative research regarding the preferred lifting method had previously been conducted, so the Israel Police Serious Crime Scene Unit treated this shoeprint as it would treat most dry origin shoeprints. The shoeprint was photographed and lifted with an adhesive lifter followed by enhancement with BPB (Fig. 2). Several accidental characteristics were observed on the shoeprint, even though there was background coloring, and the print was connected to the suspect's shoe with a high level of association.

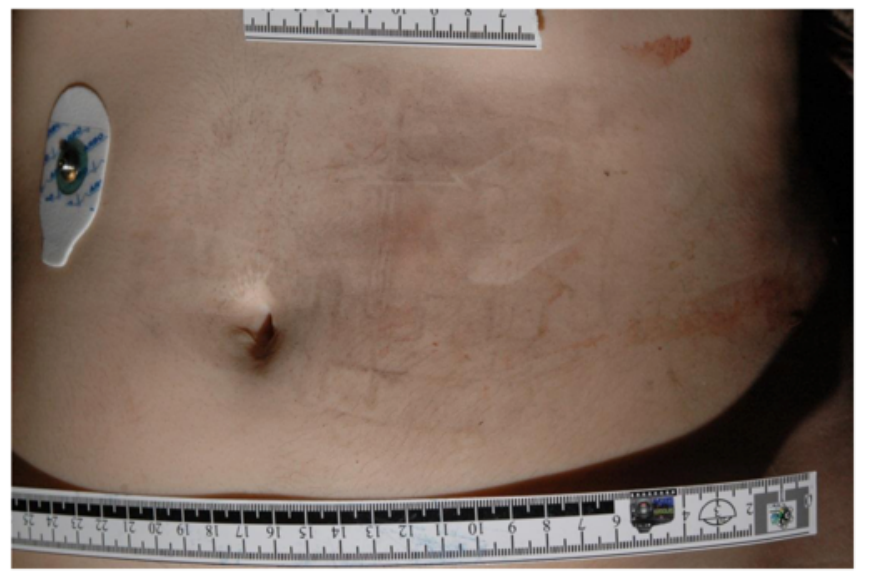

Fig. (1). Dust shoeprint on the abdomen of a murdered prostitute body.

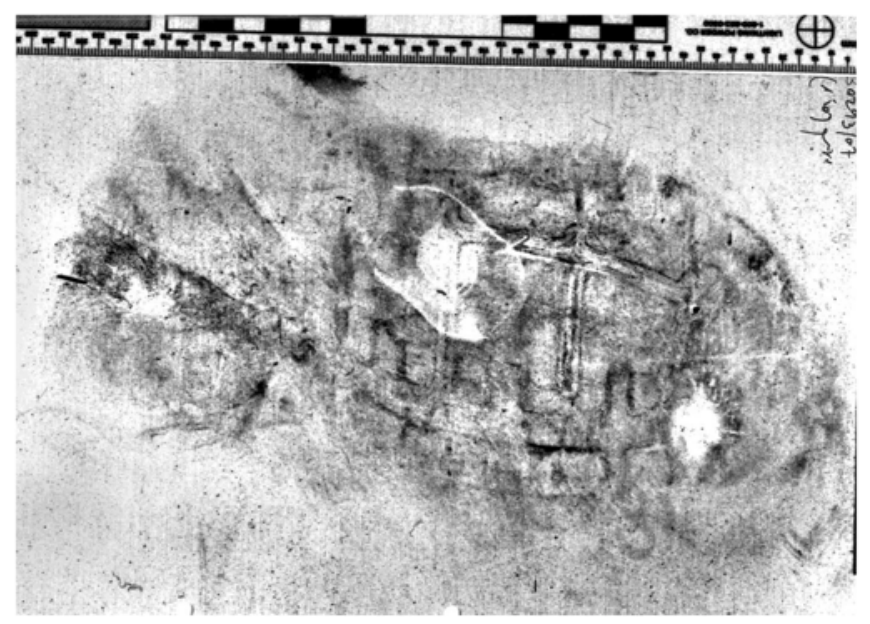

Fig. (2). The shoeprint after lifting with an adhesive lifter and enhancing with BPB.

Not all cases are so lucky. It is a general rule in fingerprint and shoeprint development that choosing the

${ }^{2}$ Carlsson, K. Comparison of lifting shoeprints with gelatine lifter versus electrostatic methods. Presentation at the Second European Meeting for SP/TM Experts; The Netherlands, 1997. appropriate developing method can determine success or failure. The human body is complexly contoured, and the incorrect development method can result in loss of details essential for the comparison process.

Shoeprints caused by blunt force trauma are more clearly visible. Several papers have been written about such cases and the optimal ways to record and observe them $[1,13]$. Papers concerning dry origin shoeprints on bodies have also been written. The method mentioned is Electrostatic lifter (ESL) $[4,14]$. It was the authors' intention to examine optimal possibilities of removing dry contaminants and methods to record them.

\section{MATERIALS AND METHODOLOGY}

To examine the possibility of removing dust shoeprints from human bodies at a crime scene an attempt was made to imitate natural stepping on a live body (not stomping). The shoe soles of a laboratory worker were cleaned by stepping on an adhesive lifter. Then the worker walked approximately fifty meters outside the laboratory. Finally, he stepped on the body of a live volunteer.

This process was repeated several times with the same person stepping on the volunteer (Fig. 3). One can, therefore, assume that all shoeprints were of similar intensity. The shoeprints were photographed according to standard protocols, once with scattered light and once with a low angel directs light.

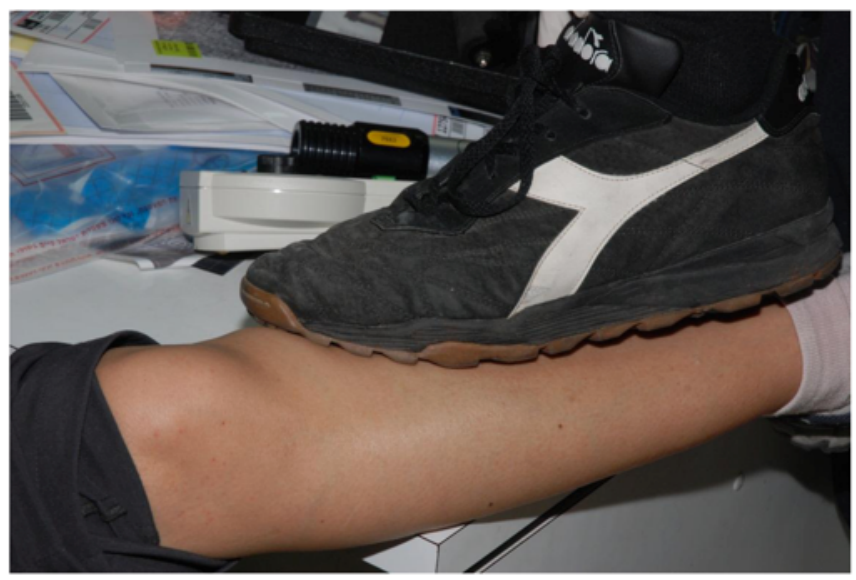

Fig. (3). Depositing the shoeprint on the leg of the volunteer by direct stepping with the shoe.

The shoeprints were then removed using three common lifting methods:

(a) Electrostatic lifter: The volunteer placed her leg on a wooden table. A black aluminum sheet was placed on the shoeprint, and a metallic plate was positioned near the mark. A generator was set to maximum voltage for a few seconds. A roller was used to adjust the strip to the curvatures of the human leg (Fig. 4).

(b) Gelatin lifter: A black gelatin lifter (BVDA, the Netherlands) [15] was pressed manually against the mark for a few seconds and then lifted (Fig. 5).

(c) Adhesive lifter followed by enhancement with BPB: A white adhesive lifter (JAC vinyl adhesive) was pressed against the mark for a few seconds (Fig. 6), 
and then it was lifted and re-covered with a silicon cover. The adhesive was sprayed with BPB solution and humidified with water vapor.

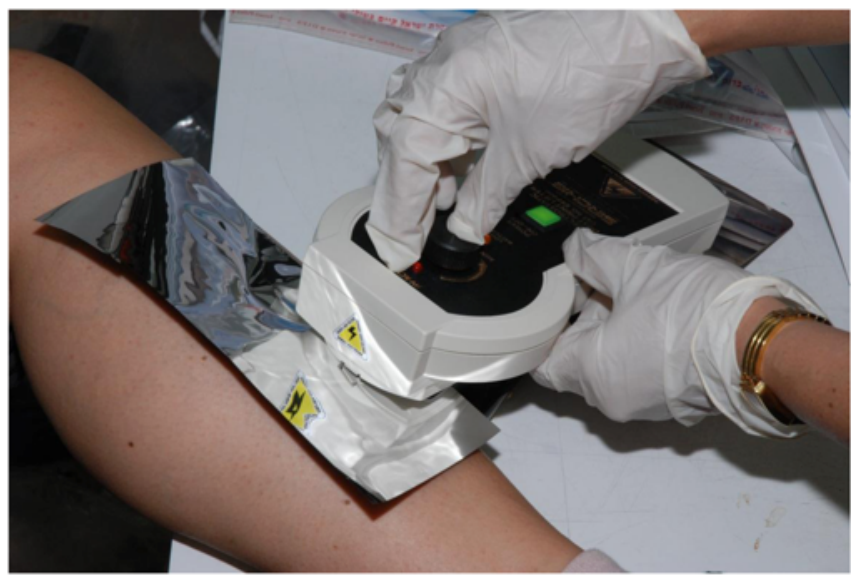

Fig. (4). Removing the dust shoeprints with ESL.

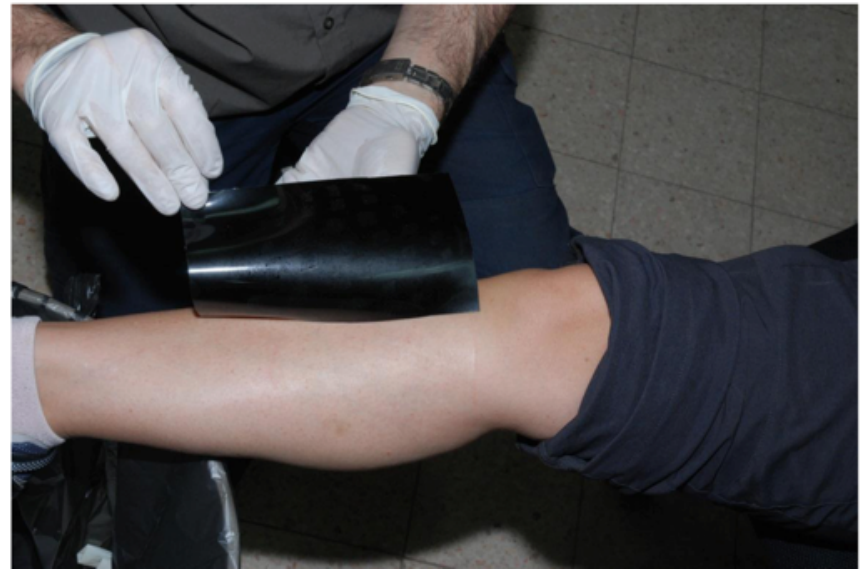

Fig. (5). Removing the dust shoeprints with a gelatin lifter.

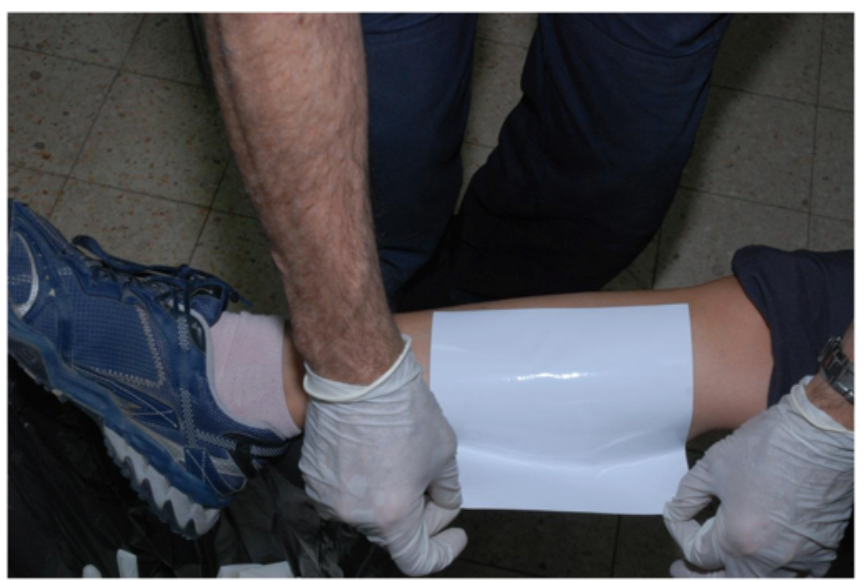

Fig. (6). Removing the dust shoeprints with an adhesive lifter.

Each developing process was tried three times.

\section{RESULTS}

The black gelatin lifter was superior to the ESL and the adhesive lifter followed by BPB in completeness of impression, sharpness of detail and contrast.
The prints lifted with black gelatin were clear and detailed (Fig. 7).

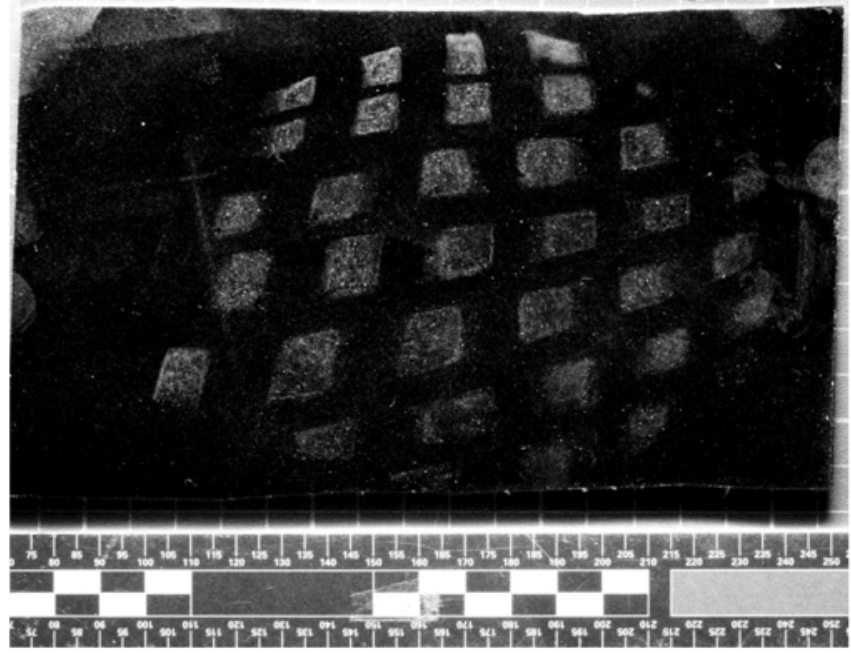

Fig. (7). Shoeprint lifted with a gelatin lifter.

Completeness of impression was reduced with the ESL due to the fact that full contact was not obtained as a result of the curved nature of the leg (Fig. 8).

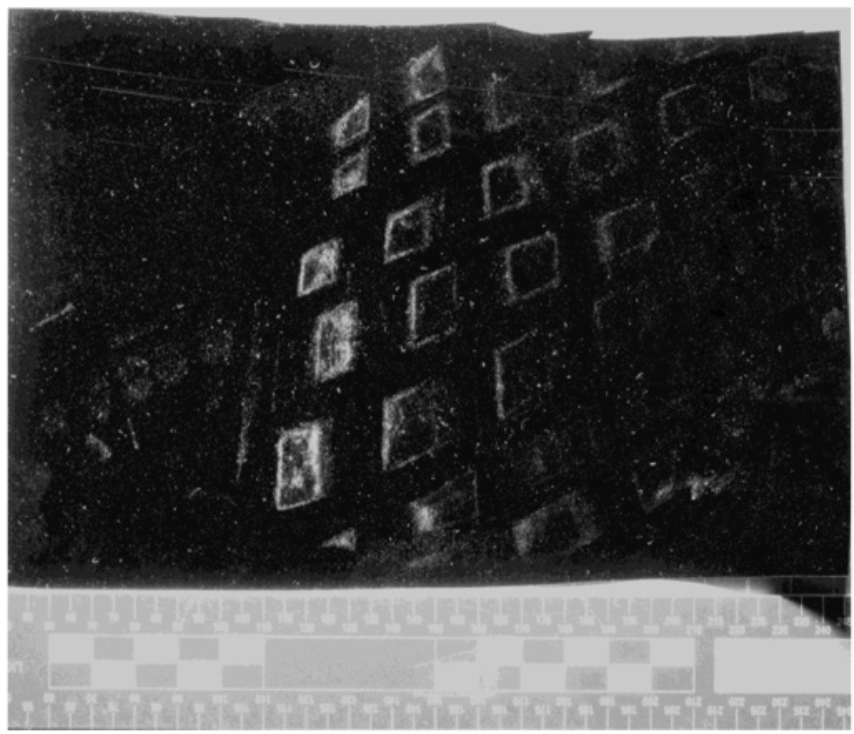

Fig. (8). Shoeprint lifted with ESL.

The BPB reacted not only with the shoeprint, but with the background as well, partially concealing the shoeprints (Fig. 9).

The detailed results are described in Table 1.

\section{DISCUSSION}

\section{ESL}

The electrostatic lifter is not a practical solution for lifting shoeprints from live bodies. Occasionally the electric charge ran through the volunteer's body to the ground, resulting in an uncomfortable feeling. Although static electricity is usually not harmful to health even with a voltage as high as $10,000 \mathrm{~V}[16,17]$, according to the ESL user manual [18] the electrical voltage (approximately 
Table 1. Comparison Between Black Gelatin Lifter, ESL and Adhesive Lifter Followed by BPB (The Prints were Graded 1-5, 5 Being the Highest Grade)

\begin{tabular}{|c|c|c|c|c|c|c|c|c|c|}
\hline \multirow[b]{2}{*}{ Quality Factor } & \multicolumn{3}{|c|}{ Black Gelatin Lifter } & \multicolumn{3}{|c|}{ ESL } & \multicolumn{3}{|c|}{ Adhesive Lifter + BPB } \\
\hline & $\begin{array}{l}\text { First } \\
\text { Trial }\end{array}$ & $\begin{array}{c}\text { Second } \\
\text { Trial }\end{array}$ & $\begin{array}{l}\text { Third } \\
\text { Trial }\end{array}$ & $\begin{array}{l}\text { First } \\
\text { Trial }\end{array}$ & $\begin{array}{c}\text { Second } \\
\text { Trial }\end{array}$ & $\begin{array}{l}\text { Third } \\
\text { Trial }\end{array}$ & $\begin{array}{l}\text { First } \\
\text { Trial }\end{array}$ & $\begin{array}{c}\text { Second } \\
\text { Trial }\end{array}$ & $\begin{array}{l}\text { Third } \\
\text { Trial }\end{array}$ \\
\hline Completeness of impression & 5 & 5 & 5 & 3 & 4 & 3 & No imprint & 4 & 2 \\
\hline Sharpness of detail & 5 & 4 & 5 & 3 & 2 & 4 & No imprint & 3 & 2 \\
\hline Contrast & 4 & 5 & 3 & 4 & 4 & 5 & No imprint & 2 & 2 \\
\hline
\end{tabular}

$10,000 \mathrm{~V})$ can be dangerous. Another disadvantage, not derived from the vitality of the volunteer, arose as well. Unlike previous research done [4, 14], it was found that full contact on curved surfaces was not easily obtained; hence, the shoeprints were not lifted entirely.

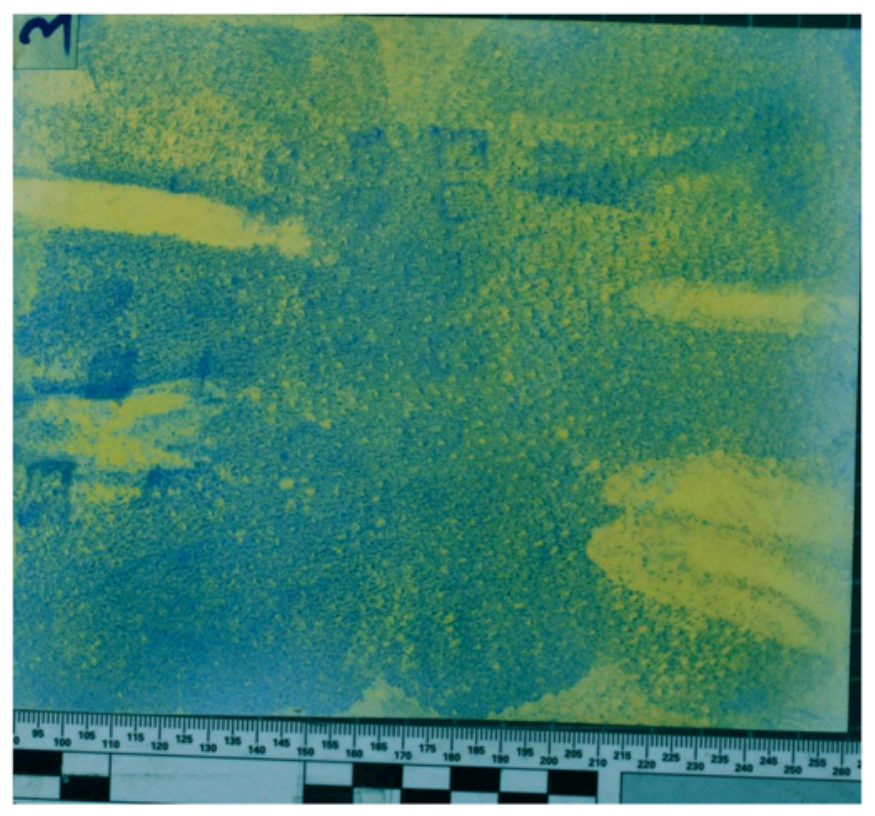

Fig. (9). Shoeprint lifted with an adhesive lifter and further developed with BPB.

\section{Adhesive Lifter Followed by BPB}

The adhesive lifter turned blue immediately after it was sprayed with BPB, concealing large sections of the shoeprints. This phenomenon was further investigated. An adhesive lifter was placed on a clean area of the volunteer's skin, lifted, then sprayed with BPB. The areas that touched the skin turned blue, and the design of the skin cells appeared. It was determined that the BPB reaction is not suitable for enhancing shoeprints lifted from human skin. The adhesive lifter removes microscopic skin particles, which adhere to the surface of the lifter. The $\mathrm{pH}$ of the human skin ranges between approximately 4.8 and 5.5, depending on the area on the body [19]. At this $\mathrm{pH}$ the BPB is in its basic form, and the skin particles are, therefore, dyed blue, revealing the pattern of the skin on the adhesive lifter. Since both the dust forming the shoeprint and the lifted skin cells turn blue, it is difficult to differentiate the shoeprint from the skin background.
There are cases, such as the one described in the introduction, in which the shoeprint contains a considerable amount of dust, and the accidental characteristics are big. In such cases the adhesive lifter followed by BPB may result in a high degree of association between the suspect shoe and the shoeprint, but in other cases with poorer shoeprints this method is not satisfactory.

\section{Black Gelatin Lifter}

The Gelatin lifter proved to be the best method for lifting dry origin shoeprints from bodies. It was successful in lifting prints even from curved surfaces due to the flexibility of the gelatin lifter.

The three methods tested are not the most desirable for shoeprints that are not of a dry origin. Shoeprints from curved surfaces, such as bodies, raise additional difficulties during the comparison process. The shoeprints tend to be distorted; hence, correspondence in size and exact design is not exact. Of course, any lifting method, including the gelatin lifter, cannot solve this.

\section{CONCLUSIONS}

The three examined procedures are commonly used for recovering dry origin shoeprints from various surfaces. In this research two of the methods were determined to be inferior to the chosen black gelatin lifter method.

With the electrostatic lifter full contact is not easily obtained especially on soft and rounded areas, and static shock may cause harm to live subjects.

Enhancement of adhesive lifters with BPB causes a background reaction that may conceal the shoeprints.

The black gelatin lifter is the best method for lifting dry origin shoeprints from bodies. It is successful in lifting prints even from curved surfaces due to the flexibility of the gelatin lifter. The prints are clear and detailed.

The experiments described in this paper were performed on live bodies. Further research on cadavers is necessary, since the results may vary. The safety hazard of using ESL on live persons is not relevant when applied to cadavers. Furthermore, the lifting of dead skin cells may behave differently when applying adhesive lifters on cadavers. On the other hand, the difficulty of attaching ESL to curved surfaces will remain the same. 


\section{CONFLICT OF INTEREST}

The author confirms that this article content has no conflicts of interest.

\section{ACKNOWLEDGEMENTS}

The authors would like to thank Prof. J. Levinson (DIFS, retired) for his editorial assistance.

\section{REFERENCES}

[1] Chochol, A.; Swietek. M. Shoe prints on the human body - an analysis of three cases. Prob. Forensic Sci., 2009, 78, 239-247.

[2] Bodziak, W.J. Footwear Impression Evidence, Detection Recovery and Examination, 2nd ed.; CRC Press: Washington D.C, 2000.

[3] Wilgus, G. Latent shoeprint recovery on human skin. J. Forensic Ident., 2004, 54(4), 428-432.

[4] Adair, T.W.; Dobersen, M. Lifting dusty shoe impressions from human skin: a review of experimental research from Colorado. $J$. Forensic Ident., 2006, 56(3), 333-338.

[5] Sampson, W.C.; Sampson, K.L. Recovery of latent prints from human skin. J. Forensic Ident., 2005, 55(3), 362-385.

[6] Lawley, R. Application of amido black mixture for the development of blood based fingerprints on human skin. $J$. Forensic Ident., 2003, 53(4), 404-408.

[7] Adair, T.W. Casting two-dimensional bloody shoe prints from concrete, fabric and human skin: A review of several methods with recommendations. International Association Bloodstain Pattern Analyst (I.A.B.P.A.) News, 2005, March, 4-8.

[8] Ojena, S.M. New electrostatic process recovers visible and invisible dust particles at crime scenes. Law Order, 1988, 3, 31-33.
[9] Hilderbrand, D.S. Footwear, the Missed Evidence, 2nd ed.; Staggs Publishing: Wildomar, CA, 2007; pp. 121-122.

[10] Shor, Y.; Vinokurov, A.; Glattstein, B. The use of an adhesive lifter and $\mathrm{pH}$ indicator for the removal and enhancement of shoeprints in dust. J. Forensic Sci., 1998, 43(1), 182-184.

[11] Wiesner, S.; Tsach, T.; Belser, C.; Shor, Y. A comparative research of two lifting methods: electrostatic lifter and gelatin lifter. $J$. Forensic Sci., 2011, 56(S1), S58-S62.

[12] Glattstein, B.; Shor, Y.; Levin, N.; Zeichner, A. pH indicators as chemical reagents for the enhancement of footwear marks. $J$. Forensic Sci., 1996, 41(1), 23-26.

[13] Park, S.; Kim, T.; Choi, Y.; Cae, S. Analysis of two tire marks on the head and clothing. J. Forensic Sci. Technol., 2006, 11(1), 125129.

[14] Tovar, R.M. The use of electrostatic equipment to retrieve impressions from the human body. J. Forensic Ident., 2004, 54(5), 530-533.

[15] BVDA International. BVDA Manual of Use for Gellifters. http://www.bvda.com/EN/download/Gellifter_brochure.pdf (accessed October 23, 2012).

[16] OSHA. Effects of Electric Curent in the Human Body. http:/www. osha.gov/Publications $/ 3075 / \mathrm{html}$ (accessed June 26, 2013).

[17] Electrical Injuries. Health and Safety Executive. http:/www.hse. gov.uk/electrcity/injuries.htm. (accessed June 26, 2013).

[18] Sirchie, fingerprint laboratories. Technial information, Electrostatic dust print lifter Catalog no. ESP900. www.sirchie.com/Asets/ Manuals/pdf/ESP900_TI01-2ENG-REV8E.pdf (accessed January 23, 2014).

[19] Yosipovitch, G.; Xong, G.L.; Haus, E.; Sackett-Lundeen, L.; Ashkenazi, I.; Mailbach, H.I. Time dependent variations of the skin barrier function in humans: transpidermal water loss, stratum corneum hydration, skin surface $\mathrm{pH}$, and skin temperature. J. Invest Dermatol., 1998, 110 (1), 20-23.

This is an open access article licensed under the terms of the Creative Commons Attribution Non-Commercial License (http://creativecommons.org/licenses/by$\mathrm{nc} / 3.0 /$ ) which permits unrestricted, non-commercial use, distribution and reproduction in any medium, provided the work is properly cited. 